\title{
On Manipulation of Objects in Three-Fingered Grasp
}

\author{
Dominique P. Chevallier*, Shahram Payandeh*(Assoc. Mem. ASME) \\ *Centre d'Enseignement et de Recherche en * Experimental Robotics Laboratory \\ Mathématiques Appliquées \\ U.R.A - C.N.R.S 1502. Central 2 \\ La Courtine \\ 93167 Noisy-le Grand Cedex, FRANCE \\ School of Engineering Science \\ Simon Fraser University \\ Burnaby, British Columbia \\ V5A 1S6, CANADA
}

\begin{abstract}
Manipulation of the grasped object is defined as the ability of the mechanical endeffector to create an instantaneous motion of the object with respect to a fixed reference frame (e.g. palm reference frame). This class of manipulation is usually referred to as the fine manipulation whereas a collection of all these instantaneous motions of the object is referred to as the gross manipulation. This paper presents a new method where for a given desired twist of the grasped object, the instantaneous motions of the finger-tips can be determined. The results of the paper are divided into two parts. First, for the case where the motion of the object is created through motions of the finger-tip in off-tangent planes to the object at the contact points. Second, where a class of motion of the grasped object is achieved through motions of the finger-tips which are restricted to the tangent planes. The method of this paper utilizes screw geometry, inner product spaces and information regarding grasp configuration. The method is also illustrated through examples.
\end{abstract}

\section{Introduction}

Grasping task using dexterous mechanical end-effector is defined as the ability of the open-loop mechanisms to make contact with the object such that the object does not slip between the fingers. Manipulation of the grasped object is further defined as the ability of the mechanical end-effector to create an instantaneous motion of the grasped object with respect to a fixed

reference frame (e.g. palm reference frame). This class of manipulation is usually referred to as the fine manipulation where a collection of all instantaneous motion of the object is referred to as gross manipulation.

Various methods have been proposed in the literature for determining the motion of the grasping fingers in order to create the desired motion of the grasped object. Hong et al (1990) presented several results on the existence of a certain three to four-fingered grasp being suitable for fine manipulation when given some constraints on the geometry of the object. Yoshikawa and Nagai (1991) presented a method for decomposing the finger-tip force into two components by using pseudo-inverse of coefficient matrix that relates the resultant force exerted on the grasped object to the fingertip force. One component is given by pre-multiplying the resultant force by the pseudo-inverse of the coefficient matrix. This component is characterized as the one with the minimum norm among those producing the specified resultant force. The other 
component is given by a vector belonging to the null space of the coefficient matrix, is an internal force. The first decomposed component is referred to as the manipulation and the other as the grasping forces. Rus (1992) suggested a strategy for dexterous manipulation called finger tracking. The reorientation of the grasped object is planned by finger tracking on the face of an object whose motion is constrained by a set of fingers fixed in space. In this way, the reorientation is accomplished by simple sliding motion of the tracking finger. Kao and Cutkosky (1992) proposed a method where manipulation of the grasped object can be accomplished by sliding action of one finger on the face of the object. The sliding trajectories are characterized by a transient and steady-state solution. By classifying the configuration of fingers grasping an object as a configuration of parallel chain manipulator, Kumar (1992) proposed a method based on screw system for obtaining transformation equations between joint coordinates of the fingers and the corresponding finger-tip displacements. For this method, it is assumed that the desired finger-tip displacements are given. Hunt, Samuel and McAree (1991) presented special configurations of multi-finger, multi-freedom grippers. Given the kinematic model of a three fingered hand where each finger has three revolute joints and using screw theory they showed that the grasped object can lose and gain freedom in certain special configurations. In their analysis for both serial and parallel manipulators, the twist of the end-point or the work-piece is obtained through inversion of a known matrix. The above paper does not address the problem of resolving the desired twist of the grasped object into the finger-tip twists which is the scope of this paper. In particular, the results of this paper can be extended to complement the results of Hunt, Samuel and McAree (1991) by not requirering the inversion of the know matrix for determining the twist of the grasped object as a function of the twists of the each finger joints.

This paper proposes a new method for determining the twist of the fingers as a function of the desired motion of the grasped object. The method is divided into two parts: a) when the motion of the finger-tip is an off-tangent plane to the object at the contact point and when the motion can be restricted to the tangent plane. The method of this paper, similar to Hong (1990), Yoshikawa and Nagai (1991) and Rus (1992) utilizes the geometrical information of the grasped configuration to determine the instantaneous motion of the finger-tips as a function of desired motion of the object. The method of this paper utilizes screw theory and reciprocal systems of screws.

The paper is organized as follow: section (2) presents preliminary definitions concerning the three fingered grasp; section (3) presents a method for determining the off-tangent plane twist of the finger-tips as a function of the desired motion of the grasped object; section (4) presents a method for determining the tangent plane motion of the finger as a function of the desired grasped motion and section (5) presents concluding remarks.

\section{Preliminaries}

This section presents preliminary definitions which are used in this paper. In this paper it is assumed that fingers are making point contact with friction and grasped objects are made of solid material. 


\subsection{Helicoidal Field}

Let $\mathbf{X}$ define a vector field (helicoidal field) $\mathbf{X}: \varepsilon \rightarrow \mathcal{E}$ such that: (Figure (1))

$$
\mathbf{X}(b)=\mathbf{X}(a)+\omega_{\mathbf{X}} \times \overrightarrow{a b}
$$

where $a$ and $b$ are any two points belonging to the tridimensional affine Euclidean space $\varepsilon$ and $\mathcal{E}$ is the underlying vector space, Chevallier (1991). This is equivalent to say that for every pair of points $(a, b)$ in $\varepsilon$, there exists a vector $\omega_{\mathbf{X}}$ such that equation $(1)$ holds. Let $\mathcal{D}$ represent such helicoidal field.

For example, when the velocity of a rigid body is described by the twist $T=(\omega ; u)$, the helicoidal velocity field $V$, is related to $T$ by:

$$
V(p)=u+\omega \times \overrightarrow{o p}
$$

where $p$ is any point in $\varepsilon$. In particular, for the origin $o$, we have: $V(o)=u$.

For a wrench $W=(f ; g)$, the associated helicoidal field $M$ (that is the moment field) is defined as:

$$
M(p)=g+f \times \overrightarrow{o p}
$$

where for the origin $o$, we have: $M(o)=g$. 
For $\mathbf{X}$ and $\mathbf{Y}$ in $\mathcal{D}$, let us define the real number:

$$
[\mathbf{X} \mid \mathbf{Y}]=\omega_{\mathbf{X}} \cdot \mathbf{Y}(p)+\omega_{\mathbf{Y}} \cdot \mathbf{X}(p)
$$

In general, the above relationship is independent of the choice of $p$ in $\varepsilon$ (The above operation is also referred to as the inner product or the Klein form of screws. We use the word inner product even though the Klein form is not positive definite. It should be pointed-out that no positive definite inner product exists in screw theory which is invariant by the Euclidean group). For example,

$$
[W \mid T]=u \cdot f+\omega \cdot g \quad \text { a real number }
$$

The relationship $[\mathbf{X} \mid \mathbf{Y}]=0$ means that the screws are reciprocal.

We further define operation $\Omega$. If $\mathbf{X} \in \mathcal{D}$, then $\Omega \mathbf{X}$ is the constant vector field given by:

$$
\Omega \mathbf{X}(p)=\omega_{\mathbf{X}} \quad \text { for all } p \in \epsilon
$$

Hence $\Omega^{2}=0$.

\subsection{Definition of Three-Fingered Grasp}

We defined three-fingered grasp by three points $\left(p_{1}, p_{2}, p_{3}\right)$ specifying the finger/contact coordinate systems and the direction of the normal to the surface of the object at the contact points (Figure 2). 


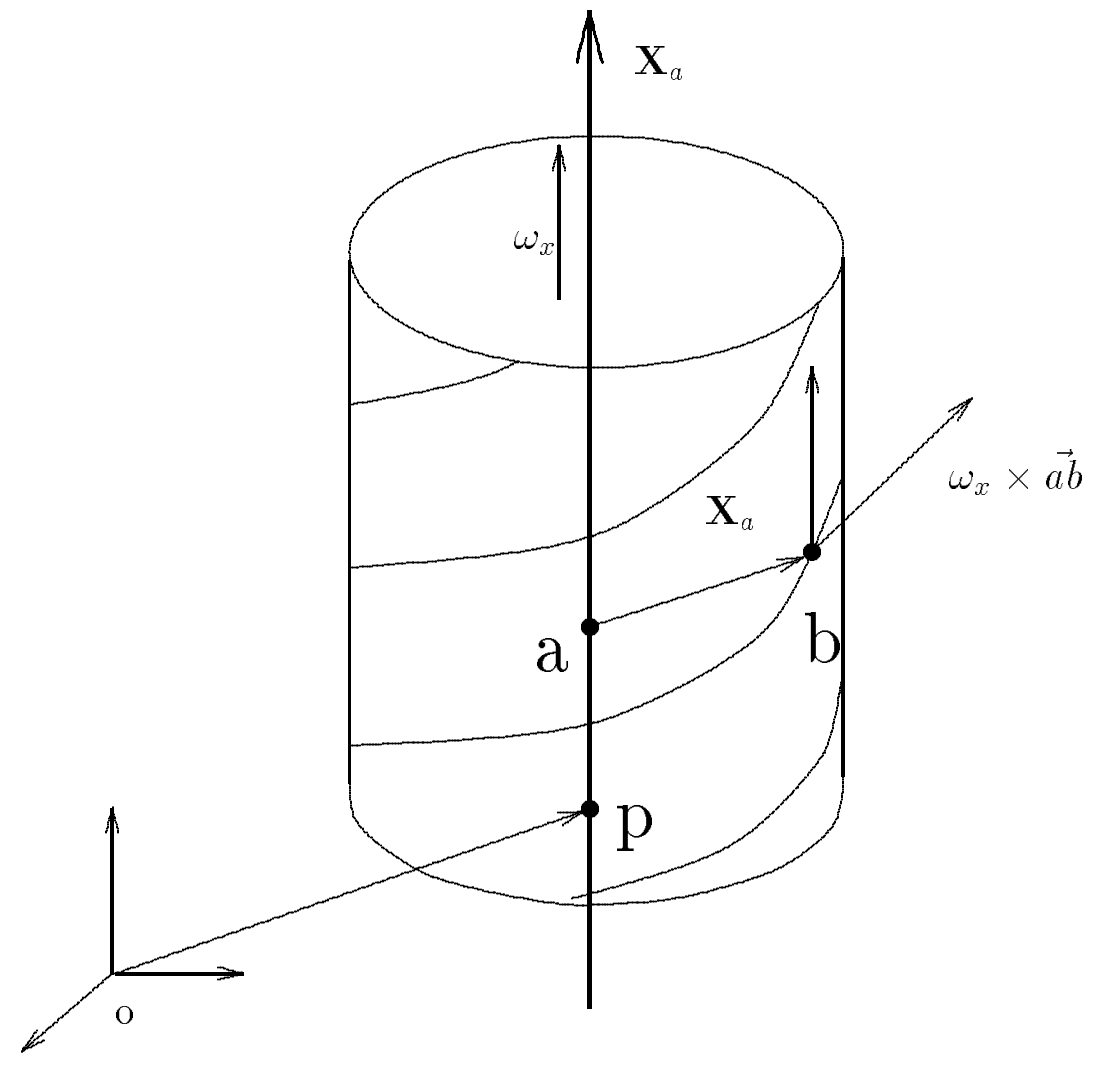

Figure 1: General definition of helicoidal field 
Along each surface normals, we define normalized screws $\nu_{1}, \nu_{2}$ and $\nu_{3}$, where for $i=1,2,3$ we have:

$$
\begin{aligned}
& {\left[\nu_{i} \mid \Omega \nu_{i}\right]=1} \\
& p_{i} \in \operatorname{axis} \text { of } \nu_{i}
\end{aligned}
$$

The screw $\nu_{i}$ describes the oriented normal to the surface of the grasped object at the point $p_{i}$. These screws are defined by screws with zero pitch. For example, the normal component of the grasping force which acts on the object at point $p_{i}$ is described by $N_{i} \nu_{i}$, where $N_{i}$ is a real number $\left(N_{i} \in \mathbf{R}\right)$ representing the intensity of the normal force.

Let $\Pi_{i}$ denote the tangential plane at $p_{i}$. Let $\mathcal{Z}_{i}$ be the vector subspace of $\mathcal{D}$ which is defined as

$$
\mathcal{Z}_{i}=\left\{\mathbf{X} \in \mathcal{D} \mid \mathbf{X}\left(p_{i}\right)=0\right\}
$$

For a finger-tip twist $T$ belonging to $\mathcal{Z}_{i}$, its axis passes through the grasping point $p_{i}$ (i.e. the value of the associated helicoidal vector field at the point $p_{i}$ is zero: $T\left(p_{i}\right)=0$ ).

In section 3.2 we shall consider the case of twists which have a moment about the point $p_{i}$ lying in the plane $\Pi_{i}$.

\section{Main Results}

The main results of this paper are divided into two parts; a) the off-tangent plane manipulation and b) tangent plane manipulation.

\subsection{Off-Tangent Plane Manipulation}

Let us define the normalized screw $\Sigma_{i}(i=1,2,3)$ as (Figure 3$)$ :

$$
\Sigma_{i}\left(p_{j}\right)=\Sigma_{i}\left(p_{k}\right)=0
$$

i.e. the axis of $\Sigma_{i}$ is the line $p_{j} p_{k}$.

The screws $\Sigma_{1}, \Sigma_{2}$ and $\Sigma_{3}$ generate a three-system of screws, that is to say a vector subspace $\mathcal{T}$ of $\mathcal{D}$ which can be defined as the set of all the elements:

$$
\alpha_{1} \Sigma_{1}+\alpha_{2} \Sigma_{2}+\alpha_{3} \Sigma_{3} \text { with } \alpha_{1}, \alpha_{2}, \alpha_{3} \in \mathbf{R}
$$

For $c \in \varepsilon$, let $\mathcal{Z}_{c}$ be the vector subspace of $\mathcal{D}$ defined by:

$$
\mathcal{Z}_{c}=\{\mathbf{X} \mid \mathbf{X}(c)=0\}
$$

and also, let us define screws $\Psi_{1}, \Psi_{2}, \Psi_{3} \in \mathcal{D}$ such that:

$$
\Psi_{i}\left(p_{i}\right)=\Psi_{i}(c)=0
$$

Let us think of the point $c$ as the center of a spherical joint (the wrist) and the points $p_{1}, p_{2}$ and $p_{3}$ as particles belonging to the grasped object (and which are moved by the finger-tips). The screw systems $\mathcal{Z}_{c}$ and $\mathcal{T}$ describe simple motions relative to the conditions of the grasp. 
A twist of the object which is described by a member of $\mathcal{Z}_{c}$ is a rotation about the point $c$ and can be produced by a motion of the wrist alone. In particular, $\Psi_{i}$ describes a rotation about the line $c p_{i}$ : the contact point $p_{i}$ remains fixed whereas $p_{j}$ and $p_{k}$ move.

A twist of the object which is described by a member of $\mathcal{T}$ is a rotation about an axis lying in the plane $p_{1} p_{2} p_{3}$ and can be produced by the motion of the fingers. In particular $\Sigma_{i}$ describes a rotation about the line $p_{j} p_{k}$ : the contact point $p_{i}$ is moved whereas the contact points $p_{j}$ and $p_{k}$ remain fixed.

The following proposition explains how any twist of the grasped object resolves into these simple motions and how to produce a given twist of the grasped object.

Proposition 1 If points $c, p_{1}, p_{2}, p_{3}$ are not in a same plane, then:

$$
\mathcal{D}=\mathcal{T} \oplus \mathcal{Z}_{c}
$$

Moreover $\left\{\Sigma_{1}, \Sigma_{2}, \Sigma_{3}, \Psi_{1}, \Psi_{2}, \Psi_{3}\right\}$ is a basis of $\mathcal{D}$ such that $\Sigma_{1}, \Sigma_{2}, \Sigma_{3} \in \mathcal{T}$ and $\Psi_{1}, \Psi_{2}, \Psi_{3} \in$ $\mathcal{Z}_{c}$.

Proof: The proof follows from the fact that for $i \neq j$ all the inner products $\left[\Sigma_{i} \mid \Sigma_{j}\right]$, $\left[\Sigma_{i} \mid \Psi_{j}\right],\left[\Psi_{i} \mid \Psi_{j}\right]$ vanish.

Let $\mathbf{T} \in \mathcal{D}$ be a twist of the grasped object. Then this twist can be expanded as:

$$
\mathbf{T}=\sum_{i=1}^{3} x_{i} \Sigma_{i}+\sum_{i=1}^{3} y_{i} \Psi_{i} \quad x_{i}, y_{i} \in \mathbf{R}
$$

where $x_{i}$ and $y_{i}$ are the magnitudes of the screws.

The corresponding amplitude of the twists about the defined basis can be obtained by forming the inner products of $\Sigma_{i}$ and $\Psi_{i}$ with both side of equation (13) respectively we can obtain:

$$
\left[\mathbf{T} \mid \Sigma_{i}\right]=y_{i}\left[\Sigma_{i} \mid \Psi_{i}\right] \quad\left[\mathbf{T} \mid \Psi_{i}\right]=x_{i}\left[\Sigma_{i} \mid \Psi_{i}\right]
$$

The assumption that $p_{1}, p_{2}, p_{3}$ and $c$ are not on the same plane implies that $\left[\Sigma_{i} \mid \Psi_{i}\right] \neq 0$ and we can solve for the amplitudes of the twists as:

$$
x_{i}=\frac{\left[\mathbf{T} \mid \Psi_{i}\right]}{\left[\Sigma_{i} \mid \Psi_{i}\right]}, \quad y_{i}=\frac{\left[\mathbf{T} \mid \Sigma_{i}\right]}{\left[\Sigma_{i} \mid \Psi_{i}\right]}
$$

Hence, the desired twist of the grasped object can be obtained as a linear combination of $\Sigma_{i}$ and $\Psi_{i}$. 
Example 1: A cylindrical object of radius 1 is grasped as is shown in the Figure. The contact points are given as: $p_{1}=(1,0,0), p_{2}=(-0.5,0.866,0$. $)$ and $p_{3}=(-0.5,-0.866,0$.$) .$ The objective is to determine the twist of each finger which can result in the desired motion of the grasped object defined by:

a) $\mathbf{T}=\Sigma_{1}$

b) $\mathbf{T}=(0,0,1 ; 0,0,0)$, (i.e.rotation about the $z-$ axis) and

c) $\mathbf{T}=(0,0,0 ; 0,0,1)$, (i.e. translation along the $z$ - axis).

From definition, the screws $\Sigma_{1}, \Sigma_{2}$ and $\Sigma_{3}$ which are normalized screws through points $\left(p_{2}, p_{3}\right),\left(p_{3}, p_{1}\right)$ and $\left(p_{1}, p_{2}\right)$ respectively are determined using the following general Plücker line coordinates representation, i.e. for two point $p_{i}=\left(x_{i}, y_{i}, z_{i}\right)$ and $p_{j}=\left(x_{j}, y_{j}, z_{j}\right)$ :

$$
\begin{aligned}
& L=x_{j}-x_{i} \\
& M=y_{j}-y_{i} \\
& N=z_{j}-z_{i} \\
& P=y_{i} N-z_{i} M \\
& Q=z_{i} L-x_{i} N \\
& R=x_{i} M-y_{i} L
\end{aligned}
$$

the normalized screw $\Sigma_{1}, \Sigma_{2}$ and $\Sigma_{3}$ can be obtained as: (screws with zero pitch)

$$
\begin{aligned}
& \Sigma_{1}=(0,-1,0 ; 0,0,0.5) \\
& \Sigma_{2}=(0.866,0.5,0 ; 0,0,0.5) \\
& \Sigma_{3}=(-0.866,0.5,0 ; 0,0,0.5)
\end{aligned}
$$

Let coordinates of point $c$ be defined as $(0,0,1)$. Based on the definition of $\Psi_{i}$, and following equation (16), we have the following description:

$$
\begin{aligned}
& \Psi_{1}=(1,0,-1 ; 0,1,0) \\
& \Psi_{2}=(-0.353,0.612,-0.707 ;-0.612,-0.353,0) \\
& \Psi_{3}=(-0.353,-0.612,-0.707 ; 0.612,-0.353,0)
\end{aligned}
$$




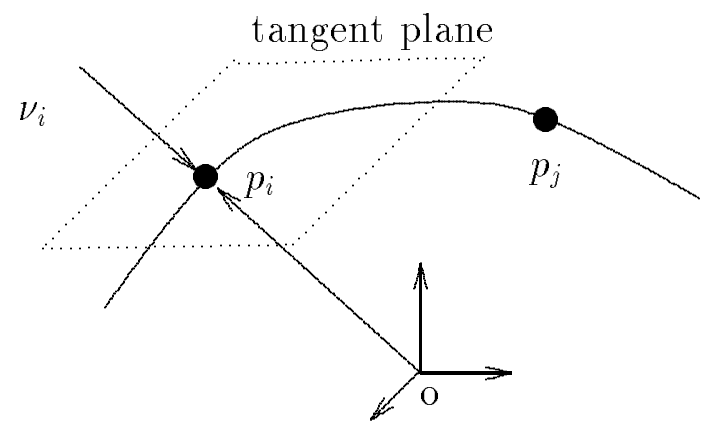

Figure 2: Definition of contact points and the oriented normals to the surface of the grasped object

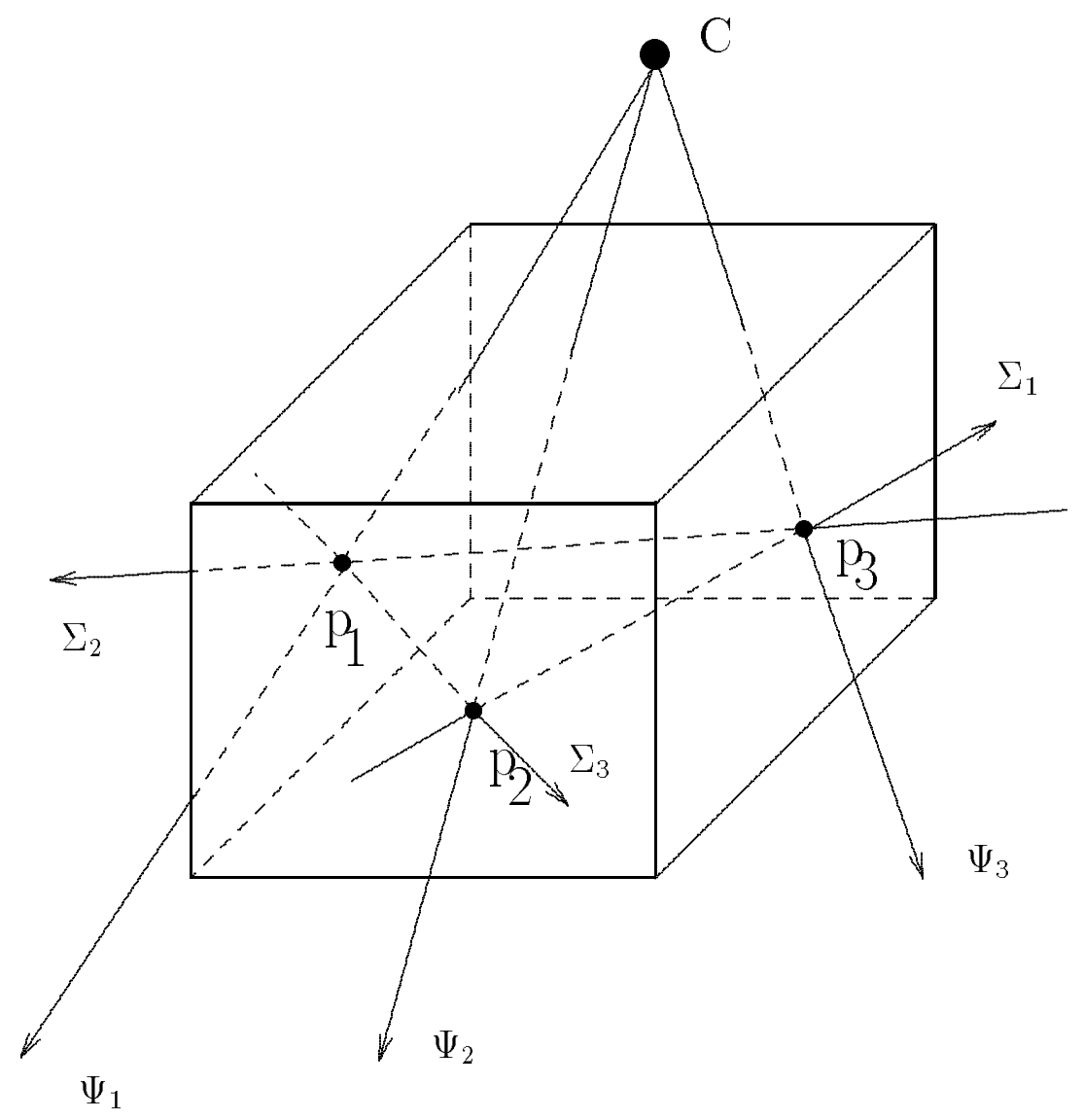

Figure 3: Definitions of $\Sigma_{i}$ and $\Psi_{i}$ in the three fingered grasp 
From these screw descriptions representing the geometry of the grasp, we have the following magnitudes for the inner products defined in equation (14):

$$
\begin{gathered}
{\left[\Sigma_{1} \mid \Psi_{1}\right]=-1.5 \quad\left[\Sigma_{1} \mid \Psi_{2}\right]=0 \quad\left[\Sigma_{1} \mid \Psi_{3}\right]=0} \\
{\left[\Sigma_{2} \mid \Psi_{1}\right]=0 \quad\left[\Sigma_{2} \mid \Psi_{2}\right]=-1.058 \quad\left[\Sigma_{2} \mid \Psi_{3}\right]=0} \\
{\left[\Sigma_{3} \mid \Psi_{1}\right]=0 \quad\left[\Sigma_{3} \mid \Psi_{2}\right]=0 \quad\left[\Sigma_{3} \mid \Psi_{3}\right]=-1.058}
\end{gathered}
$$

For case a), we have the following decomposition of the desired twist $\mathbf{T}$ into its components (i.e. the trivial case). For this case, $\left[\mathbf{T} \mid \Sigma_{i}\right]=0$ and $\left[\mathbf{T} \mid \Psi_{i}\right]=0$ for $i=2,3$ and $\left[\mathbf{T} \mid \Psi_{1}\right]=-1.5$ . As a results, we have:

$$
\mathbf{T}=(0,-1,0 ; 0,0,0.5)
$$

Figure (5), shows the schematics of the manipulation of the object for this case. In this example, since the axis of twist is $\Sigma_{1}$, the contact point $p_{1}$ has instantaneous rotation in a circular path about the axis $p_{2} p_{3}$. 
For the case $\mathrm{b})$, the desired twist of the object is defined as the rotation about the $z-$ axis or $\mathbf{T}=(0,0,1 ; 0,0,0)$. Here, the nonzero inner products are calculated to be:

$$
\left[\mathbf{T} \mid \Sigma_{1}\right]=\left[\mathbf{T} \mid \Sigma_{2}\right]=\left[\mathbf{T} \mid \Sigma_{3}\right]=0.5
$$

Following equation (13), the desired twist of the object can be obtained by the sum of the twist of each finger-tips, or

$$
\mathbf{T}=y_{1} \Psi_{1}+y_{2} \Psi_{2}+y_{3} \Psi_{3}
$$

where $y_{1}=-0.333, y_{2}=-0.472$ and $y_{3}=0.472$ are the amplitudes of the twists about $\Psi_{1}, \Psi_{2}$ and $\Psi_{3}$.

Figure (6) shows an example of accomplishing the desired twist of the object $\mathbf{T}$ by sequentially holding finger $i$ fixed while moving the fingers $j$ and $k$ such that the final desired twist of the object is accomplished. Another alternative would be to rotate the wrist without moving the fingers.

To verify the expansion we can write:

$$
\begin{aligned}
\mathbf{T}= & -0.333(1,0,-1 ; 0,1,0)-0.472(-0.353,0.612,-0.707 ;-0.612,-0.353,0) \\
& -0.472(-0.353,-0.612,-0.707 ; 0.612,-0.353,0) \\
= & (0,0,1 ; 0,0,0)
\end{aligned}
$$




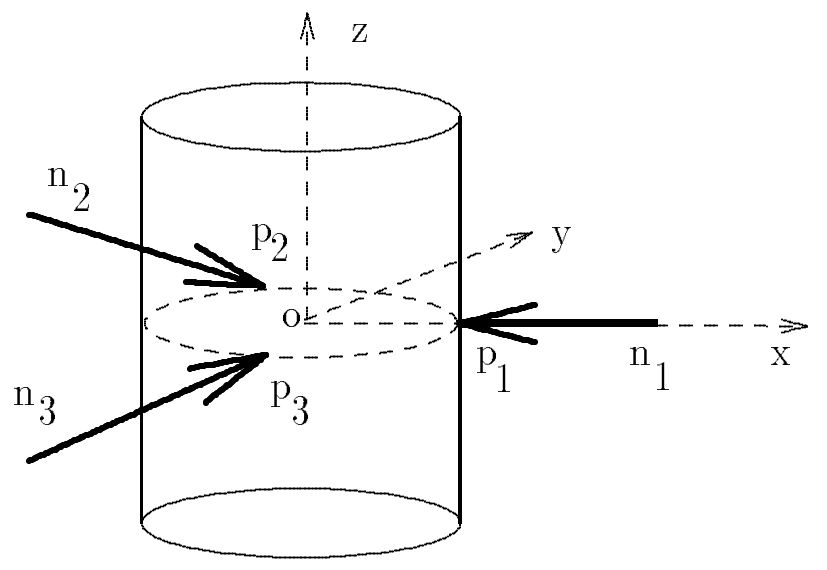

Figure 4: The grasp configuration of Example (1)

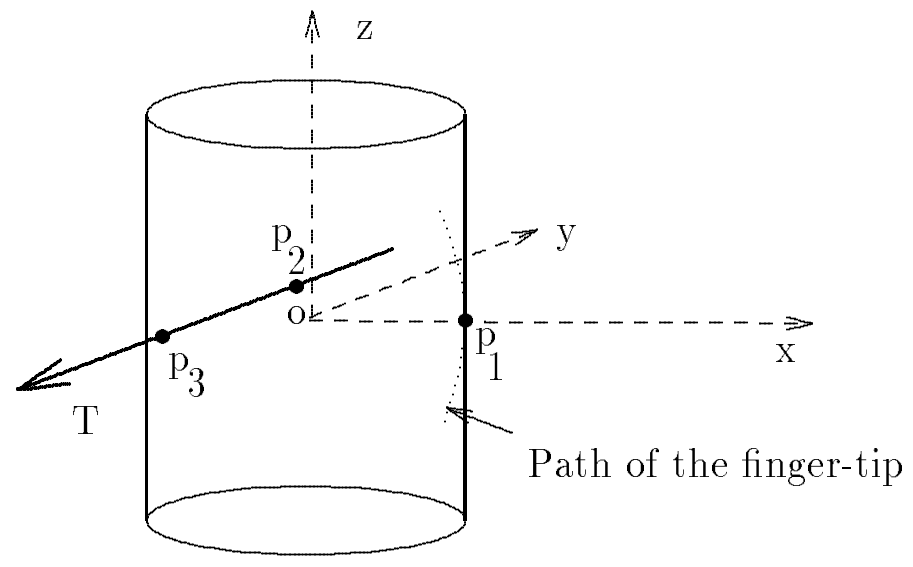

Figure 5: Schematics of fine manipulation of Example 1a 
For the case $\mathrm{c}$ ) where the desired twist of the object is given as translation along the $z-$ axis or : $\mathbf{T}=(0,0,0 ; 0,0,1)$. For this case, $\left[\mathbf{T} \mid \Sigma_{i}\right]=0$ for $i=1,2,3$ and we have:

$$
\left[\mathbf{T} \mid \Psi_{1}\right]=\left[\mathbf{T} \mid \Psi_{2}\right]=\left[\mathbf{T} \mid \Psi_{3}\right]=0.668
$$

As a result, the desired twist of the object can be obtained by sum of the twists about $\Sigma_{1}, \Sigma_{2}$ and $\Sigma_{3}$. Or,

$$
\begin{aligned}
\mathbf{T}= & 0.666(0,-1,0 ; 0,0,0.5)+0.666(0.866,0.5,0 ; 0,0,0.5) \\
& 0.668(-0.866,0.5,0 ; 0,0,0.5) \\
\text { or }= & (0,0,0 ; 0,0,1)
\end{aligned}
$$

\subsection{Tangent Plane Manipulation}

Previous subsection presented a method for determining the desired twist of the grasped object by the sum of the twist of each finger-tip. The results were obtained by assuming that there exist no restriction on the motion of each finger-tip and they can be in any off-tangent planes. In some cases it may be required to restrict the motion of each finger to the plane which is tangent to the object surface at the contact points.

This section presents a method for resolving the desired twist of the object into the sum of the three twists such that their respective values at the grasping points $p_{1}, p_{2}$ and $p_{3}$ are directed as the tangential planes $\Pi_{1}, \Pi_{2}$ and $\Pi_{3}$. It is noteworthy that in such manipulation, beside lying in the planes $\Pi_{1}, \Pi_{2}$ and $\Pi_{3}$, the velocities of the finger-tips must agree with the rigidity of the grasped object and their values can not be set arbitrarily.

Let $\mathcal{U}$ be the vector subspace of $\mathcal{D}$ defined by:

$$
\mathbf{T} \in \mathcal{U} \Longleftrightarrow \mathbf{T} \in \mathcal{D} \quad \text { and } \mathbf{T}\left(p_{i}\right) \text { is directed as } \Pi_{i} \text { for } i=1,2,3
$$

For a given twist of the grasped object $\mathbf{T}$, the component of the twist which makes a motion along the normal to the tangent surface at $p_{i}$ can be written as:

$$
\left[\mathbf{T} \mid \nu_{i}\right]=\mathbf{T}\left(p_{i}\right) \cdot n_{i}
$$

where $n_{i}$ is the normal vector to the tangent plane. Hence, twist inducing tangent velocities at $p_{1}, p_{2}, p_{3}$ make a three-system of screws defined by:

$$
\mathbf{T} \in \mathcal{U} \Longleftrightarrow \mathbf{T} \in \mathcal{D} \text { and }\left[\mathbf{T} \mid \nu_{i}\right]=0 \text { for } i=1,2,3
$$

In other words $\mathcal{U}$ is the system of screws which are reciprocal to the three screws $\nu_{1}, \nu_{2}$ and $\nu_{3}$. If the rank of the $\left\{\nu_{1}, \nu_{2}, \nu_{3}\right\}$ is three the dimension of $\mathcal{U}$ is also three.

Proposition 2 If the rank of $\left\{\nu_{1}, \nu_{2}, \nu_{3}\right\}$ is three, there exists $\Theta_{1}, \Theta_{2}, \Theta_{3} \in \mathcal{D}$ with the following properties:

1. $\left(\Theta_{1}, \Theta_{2}, \Theta_{3}\right)$ is a basis of $\mathcal{U}$

D. $\Theta_{i} \in \mathcal{Z}_{i}$ for $i=1,2,3$. 


\section{Proof:}

To prove the existence of $\Theta_{i}$, let us calculate $\omega_{i}$ the invariant vector of $\Theta_{i}$. We have:

$$
\Theta_{i}\left(p_{j}\right)=\omega_{i} \times p_{i} \vec{p}_{j}, \quad \Theta_{i}\left(p_{k}\right)=\omega_{i} \times p_{i} \vec{p}_{k}
$$

The above condition state that $\Theta_{i} \in \mathcal{Z}_{i}$. In addition from (20) we can deduce that:

$$
\begin{aligned}
& \left.\left(\omega_{i} \times p_{i} \vec{p}_{j}\right) \cdot n_{j}=\omega_{i} \cdot\left(\left(p_{i} \vec{p}_{j}\right) \times n_{j}\right)\right)=0 \\
& \left.\left(\omega_{i} \times p_{i} \vec{p}_{k}\right) \cdot n_{k}=\omega_{i} \cdot\left(\left(p_{i} \vec{p}_{k}\right) \times n_{k}\right)\right)=0
\end{aligned}
$$

A solution is:

$$
\omega_{i}=\left(n_{j} \times p_{i} \vec{p}_{j}\right) \times\left(n_{k} \times p_{i} \vec{p}_{k}\right)
$$

Example 2: Figure (7) shows three points contact between a dexterous mechanical endeffector and a polyhedral object. The objective is to give the object a twist with a unit amplitude with respect to the coordinate frame of the object defined as: $\mathbf{T}=(0.577,0.577,0.577 ; 0.0,0.0,0.0)$. The constrained on the manipulation however is that the instantaneous motions of the fingertips are restricted to their corresponding tangent planes. 
From the geometry of the grasp configuration, the contact point coordinates and the corresponding unit normals to the tangent planes can be written as:

$$
\begin{array}{lll}
p_{1}=(0,2,2) & ; & n_{1}=(1,0,0) \\
p_{2}=(2,0,2) & ; & n_{2}=(0,1,0) \\
p_{3}=(2,2,0) & ; & n_{3}=(0,0,1)
\end{array}
$$

Following Proposition 2, we can see that the rank condition for the grasp configuration is satisfied. To solve for the invariance of the twist axis $\omega_{i}$, from Equation 2 we have, for example, $p_{1} \vec{p}_{2}=(2 i, 0 j,-2 k)$ and $\vec{p}_{1} \vec{p}_{2}=(2 i,-2 j, 0 k)$. Then we have: $\omega_{1}=\left(n_{2} \times p_{1} \vec{p}_{2}\right) \times\left(n_{3} \times p_{1} \vec{p}_{3}\right)$ which results in $\omega_{1}=(4 i, 0 j, 0 k)$. Similarly we can obtain $\omega_{2}=(0 i, j, 0 k)$ and $\omega_{3}=(0 i, 0 j, k)$. Having $\omega_{i}$ and a point on the its axis, namely $p_{i}$, from the line coordinates defined in Equation 15, we can determine the coordinate of the twist axis $\Theta_{i}$ as follows:

$$
\begin{aligned}
& \Theta_{1}=(1,0,0 ; 0,2,2) \\
& \Theta_{2}=(0,1,0 ;-2,0,2) \\
& \Theta_{3}=(0,0,1 ; 2,-2,0)
\end{aligned}
$$

From the grasp configuration, the coordinates of the screws $\Sigma_{1}, \Sigma_{2}$ and $\Sigma_{3}$ can be determined as (note: $\left.\Sigma_{i}\left(p_{j}\right)=\Sigma_{i}\left(p_{k}\right)=0\right)$ :

$$
\begin{aligned}
& \Sigma_{1}=(0,0.707,0.707 ;-1.414,1.414,1.414) \\
& \Sigma_{2}=(-0.707,0.0,0.707 ; 1.414,-1.414,1.414) \\
& \Sigma_{3}=(0.707,-0.707,0.0 ; 1.414,1.414,-1.414)
\end{aligned}
$$

From proposition 2 we have the following expansion between the desired twist of the object and the basis $\Theta_{i}$, or:

$$
\mathbf{T}=\alpha_{1} \Theta_{1}+\alpha_{2} \Theta_{2}+\alpha_{3} \Theta_{3}
$$

By definition, the following relationships exists:

$$
\left[\Theta_{i} \mid \Sigma_{j}\right]=\left[\Theta_{i} \mid \Sigma_{k}\right]=0 \quad \text { for } i \neq j, k
$$

Forming the inner product of the screw $\Sigma_{i}$ with both sides of equation (25) and solving for the amplitudes $\alpha_{i}$ of the twist along $\Theta_{i}$, we have:

$$
\begin{aligned}
& \alpha_{1}=\frac{\left[\mathbf{T} \mid \Sigma_{1}\right]}{\left[\Theta_{1} \mid \Sigma_{1}\right]} \\
& \alpha_{2}=\frac{\left[\mathbf{T} \mid \Sigma_{2}\right]}{\left[\Theta_{2} \mid \Sigma_{2}\right]} \\
& \alpha_{3}=\frac{\left[\mathbf{T} \mid \Sigma_{3}\right]}{\left[\Theta_{3} \mid \Sigma_{3}\right]}
\end{aligned}
$$

or,

$$
\alpha_{1}=0.577 \quad \alpha_{2}=0.577 \quad \alpha_{3}=0.577
$$

The tangent plane manipulation can be accomplished using two approaches. One is that each finger-tip at their points of contact with the object create a twist $\Theta_{i}$ with the amplitude $\alpha_{i}$. Second approach can be that for each contact point, the resultant velocity can be resolved by $\left(\Theta_{1}\left(p_{2}\right), \Theta_{3}\left(p_{2}\right)\right),\left(\Theta_{2}\left(p_{1}\right), \Theta_{3}\left(p_{1}\right)\right)$ and $\left(\Theta_{1}\left(p_{3}\right), \Theta_{2}\left(p_{3}\right)\right)$. These resultant motions at each contact point can then be created by each finger-tip which by the Proposition 2 are in the tangent planes. 


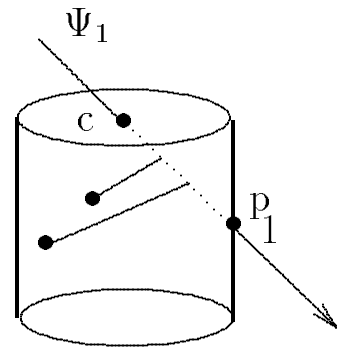

a)

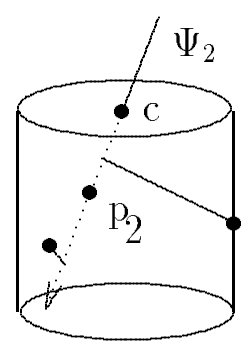

b)

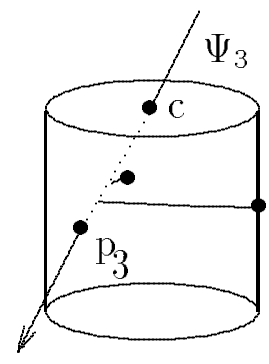

c)

Figure 6: Schematics of fine manipulation of Example 1b. a) is the twist about $\Psi_{1}$ by magnitude of $\left.y_{1}, \mathrm{~b}\right)$ is a twist about $\Psi_{2}$ by magnitude of $y_{2}$ and finally c) is the twist about $\Psi_{3}$ by $y_{3}$.

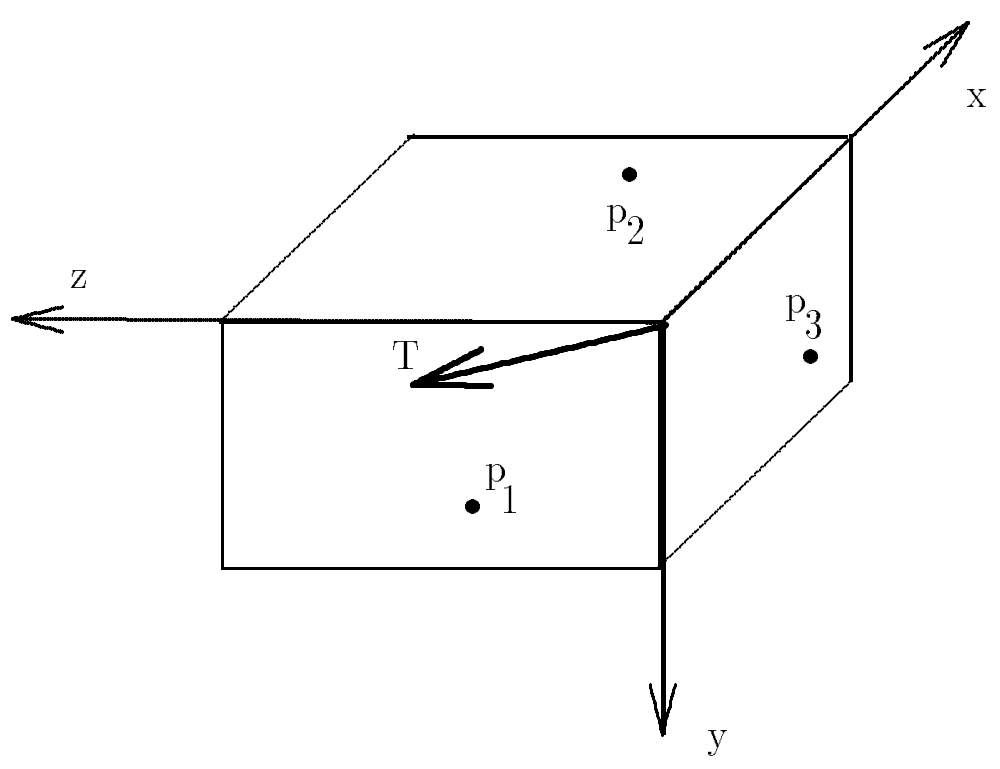

Figure 7: Schematics of the grasp configuration of Example 2 


\section{Conclusion}

Establishing a secure grasp such that the object can not slip between fingers of a dexterous mechanical end-effector is one of the main requirements in robotic pick-and-place operations. In addition to this requirement, in most cases it may also be necessary for the fingers to displace or reorient the grasped object for accomplishing a given task.

In this paper, a method for resolving the desired twist of the grasped object into the instantaneous motions of the finger-tips is presented. The results for both tangent and off-tangent planes manipulation of the grasped object at the contact points are presented.

The method of this paper offer an elegant and effective method for determining the motions of the finger-tips as a function of the grasp geometry. The method utilizes screw geometry, linear vector and the inner product spaces. The results of this paper are demonstrated through two examples.

\section{$5 \quad$ References}

Hong, J, Lafferriere, G, Mishra, B, and Tan, X,Fine Manipulation with Multifingered Hands, Proceedings of IEEE International Conference on Robotics and Automation, 1990, pp. 15681573

Yoshikawa, T. and Nagai, K.,Manipulating and Grasping Forces in Manipulation by Multifingered Robot Hands, IEEE Transaction on Robotics and Automation, Vol. 7, No. 1, 1991, pp. $67-77$

Rus, D., Dexterous Rotations of Polyhedra, Proceedings of IEEE International Conference on Robotics and Automation, 1992, pp. 2758-2763

Kao, I. and Cutkosky, M.,Quasistatic Manipulation with Compliance and Sliding, The International Journal of Robotics Research, Vol. 11, No. 1, 1992, pp. 20-40

Kumar, V.,Instantaneous Kinematics of Parallel-Chain Robotic Mechanisms, ASME Journal of Mechanical Design, Vol. 114, 1992, pp. 349-358

Hunt, K.H., Samuel, A.E. and McAree, P.R.,Special Configurations of Multi-finger Multifreedom Grippers-A Kinematic Study, The International Journal of Robotics Research, Vol. 10, No.2, April 1991, pp. 123-134

Chevallier, D.Lie Algebra, Modules, Dual Quaternions and Algebraic Methods in Kinematics, Mechanisms and Machine Theory, Vol. 26(2), pp. 613-627 